\title{
Experimentelle Modelle für räumlichen Neglect (Studien in humanen und nicht-humanen Primaten)
}

\author{
Melanie Wilke, Peter Dechent und Carsten Schmidt-Samoa
}

\begin{abstract}
Zusammenfassung
Räumlicher Neglect stellt eine schwerwiegende Konsequenz von Hirnläsionen dar und ist durch die perzeptuelle und motorische Vernachlässigung einer Raumhälfte gekennzeichnet. Die zu Neglect führenden Läsionen treten innerhalb verteilter Netzwerke auf, die sich sowohl aus kortikalen Arealen (z.B. fronto-parietalen) als auch aus subkortikalen Strukturen (z.B. dem thalamischen Pulvinar) zusammensetzen. Die Entwicklung effektiver therapeutischer Strategien setzt ein detailliertes Verständnis der wichtigen Knotenpunkte und ihrer Interaktionen voraus. Allerdings sind präzise Informationen dazu aus Patientenstudien, aufgrund der Größe und Variabilität der mit Neglect assoziierten Läsionen, oft nur schwer zu erhalten. Experimentelle Modelle, bei denen definierte Gehirnregionen systematisch inaktiviert werden können, stellen daher eine wichtige Ergänzung zu den klassischen Läsionsstudien dar. Neglect-Modelle wurden beispielsweise bei nicht-humanen Primaten mithilfe lokaler, reversibler pharmakologischer Inaktivierung entwickelt, sowie bei gesunden Probanden mithilfe nicht-invasiver Stimulations-/Inaktivierungsmethoden wie der transkraniellen Magnetstimulation. In diesem Artikel diskutieren wir Theorien zum räumlichen Neglect, insbesondere die des hemisphärischen Ungleichgewichtes, und ihre empirische Evidenz. Ein Fokus liegt dabei auf Ergebnissen aus funktionellen Bildgebungsstudien, welche die Auswirkungen lokaler Läsionen auf dynamische Netzwerkaktivität untersuchen.
\end{abstract}

\begin{abstract}
Spatial neglect is a debilitating neuropsychological disorder that is characterized by an impaired or lost ability to explore the space contralateral to the lesion and to react to stimuli presented on this side. Lesion sites that have been implicated in spatial neglect form a widely distributed network consisting of a number of cortical (i.e. fronto-parietal) and subcortical (i.e. thalamic) areas which are in healthy individuals activated during attentional and visuomotor tasks. While a detailed understanding of the brain circuits and mechanisms involved in spatial neglect is a prerequisite for the development of effective therapies, this has proven to be difficult in human patients, partly due to the extent and variability between lesion sites. Therefore, experimental models where pre-defined brain regions can be systematically inactivated are of great advantage. Neglect models have been developed in non-human primates where it is possible to pharmacologically inactivate small brain regions and in humans by means of non-invasive stimulation/ inactivation methods such as transcranial magnetic stimulation. In this paper, we discuss theories about the mechanisms of spatial neglect such as the hemispheric imbalance model and the supporting experimental evidence, with an emphasis on imaging experiments that combined lesions with measures of dynamic brain activity.
\end{abstract}

Keywords: spatial neglect; pulvinar; eye movements; visual awareness; attention

\section{Einführung}

Die Fähigkeit, uns auf bestimmte Aspekte der Umwelt zu konzentrieren und strategisch zwischen verschiedenen Handlungsoptionen $\mathrm{zu}$ entscheiden, ist eine bedeutende kognitive Leistung. Die räumliche
Ausrichtung der Aufmerksamkeit bestimmt dabei, welche sensorischen Stimuli in unser Bewusstsein gelangen, wohin wir unsere Augen ausrichten und wie wir uns im Raum bewegen. Die Erforschung der neuronalen Grundlagen normaler und gestörter räumlicher Verarbeitungsprozesse hat in den letzten beiden Jahrzehnten große Fortschritte gemacht, vor allem durch den Einsatz von nicht-invasiven bildgebenden Verfahren wie der funktionellen MRT (fMRT, siehe Exkurs 1). Während fMRTStudien viel zum Verständnis der funktionalen Komponenten der Raumkognition beigetragen haben, stammt das detaillierteste Wissen aus elektrophysiologischen Ableitungen von einzelnen Neuronen beim Affen, während diese visuelle Aufmerksamkeits- und Bewegungsaufgaben ausführen.

$\mathrm{Zu}$ den Gehirnstrukturen, die sowohl beim Menschen als auch beim Affen bei der Ausführung räumlicher Aufgaben aktiviert werden, gehören der Frontalkortex (z.B. die frontalen Augenfelder (FEF), der dorsolaterale präfrontale Kortex (dlPFC)), der obere Temporallappen (Gyrus temporalis superior), der posteriore Parietalkortex sowie eine Reihe subkortikaler Strukturen, die mit diesen kortikalen Arealen anatomisch verbunden sind (Superiorer Colliculus (SC), Putamen, Nucleus caudatus und das Pulvinar). Diese Strukturen bilden ein verzweigtes Netzwerk spezialisierter Areale und obwohl noch nicht bis ins letzte Detail erforscht, finden sich zahlreiche funktionale Homologien zwischen Menschen und Affen (Grefkes und Fink 2005; Kagan et al. 2010).

Während korrelative Ansätze am gesunden Gehirn wichtige Einblicke in die Funktionsweise des gesamten Netzwerkes geben, lässt sich dabei nicht feststellen, welche der Areale für eine bestimmte Funktion wirklich notwendig sind. Läsionsstudien stellen daher eine wichtige Ergänzung dar. In klassischen Läsionsstudien werden die Auswirkungen struktureller Läsionen mit Verhaltensdefiziten korreliert, wobei sich die Möglichkeiten zur Lokalisation von Schädigungen in der grauen Substanz und der Faserverbindungen mit der Einführung moderner MRT-Verfahren stetig verbessert haben. Die Auswirkungen struktureller Läsionen lassen sich beim Menschen beispielsweise nach dem Auftreten von Schlaganfällen studieren, während beim Affen umgrenzte Läsionen unter experimentell kontrollierten Bedingungen induziert werden können. Eine Alternative zu den strukturellen Läsionsmethoden stellen inzwischen die reversiblen Läsionsverfahren dar, mit deren Hilfe die Aktivität in bestimmten Gehirnregionen zeitweise ausgeschaltet werden kann. Beim Menschen wird dazu meist die nicht-invasive transkranielle Magnetstimulation (TMS, siehe Exkurs 3) verwendet, während beim Affen auch invasive und anatomisch 


\section{Exkurs 1}

\section{fMRT}

Die Magnetresonanz-Tomografie (MRT) ist heute eines der wichtigsten Verfahren in der diagnostischen Bildgebung. Durch die Kombination eines starken statischen Magnetfeldes (etwa das 60000-fache des Erdmagnetfeldes), kurzen HochfrequenzImpulsen im UKW-Bereich und magnetischen Zusatzfeldern gelingt es, nicht-invasiv in wenigen Minuten die Struktur des Gehirns darzustellen. Die MRT zeichnet sich durch einen exzellenten Weichgewebekontrast sowie eine hohe Sensitivität für pathologische Veränderungen aus. Für die neurowissenschaftliche Forschung von besonderem Interesse ist die funktionelle MRT (fMRT) zur Kartierung von Hirnaktivitätsänderungen. Hierbei werden die Messparameter so eingestellt, dass die lokale Signalintensität im Bild sehr stark von der lokalen Magnetfeldhomogenität abhängt. Im Rahmen einer dynamischen fMRT-Untersuchung wird die lokale Magnetfeldhomogenität hauptsächlich durch die dynamische Veränderung der lokal vorhandenen Konzentration von Deoxyhämoglobin bestimmt. Im Gegensatz zum diamagentischen Oxyhämoglobin besitzt Deoxyhämoglobin paramagnetische Eigenschaften, führt zu lokalen Magnetfeldinhomogenitäten und beeinflusst somit das MRT-Signal negativ - der sogenannte blood oxygenation level dependent (BOLD) Effekt.

Dieser Effekt kann für die Kartierung kortikaler Systeme und Netzwerke von der primären Sensorik bis zur kognitiven Informationsverarbeitung genutzt werden. Kommt es im Zuge einer Aufgabe bei der Versuchsperson zu einer veränderten (gesteigerten) synaptischen Aktivität der Nervenzellen, so resultiert dies lokal unter anderem in einem Anstieg des Blutflusses und in einer Überversorgung mit oxygeniertem Blut (Oxyhämoglobin). Das Überangebot von Oxyhämoglobin führt zu einem Auswaschen von venösem deoxygeniertem Blut (Deoxyhämoglobin). Das Auswaschen des Deoxyhämoglobins in Assoziation mit der gesteigerten neuronalen Aktivität resultiert also in einer lokal verbesserten Magnetfeldhomogenität und damit in einem Anstieg des BOLD-Signals im Bild. Die fMRT erlaubt somit über die neurovaskuläre Kopplung, indirekt auf die zugrunde liegende Hirnaktivitätsänderung zu schließen.
Auf diesem Prinzip beruhend kann die fMRT mit verschiedenen Aufgaben durchgeführt werden. Die fMRT kann aber auch genutzt werden, um die ,Ruheaktivität" des Gehirns zu erfassen und in Kombination mit einer funktionellen Konnektivitätsanalyse Regionen zu identifizieren, die zu verschiedenen (sub)kortikalen Netzwerken gehören. In der Datenanalyse werden dann die BOLD-Signalfluktuationen einzelner Hirnregionen miteinander verglichen und eine entsprechend hohe Korrelation im Sinne der Zugehörigkeit zu einem gemeinsamen Netz- werk interpretiert. Ein häufig eingesetztes Analyseverfahren ist beispielsweise die „Seed Region“-Analyse, in deren Rahmen zunächst ein BOLD-Signalzeitverlauf aus einer Region extrahiert wird, welcher dann mit den Zeitverläufen anderer Regionen korreliert wird. Mit der funktionellen Konnektivitätsanalyse können neben den verschiedenen sensomotorischen Netzwerken auch Netzwerke höherer kognitiver Funktionen, wie beispielsweise das dorsale und ventrale Aufmerksamkeitsnetzwerk, dargestellt werden.

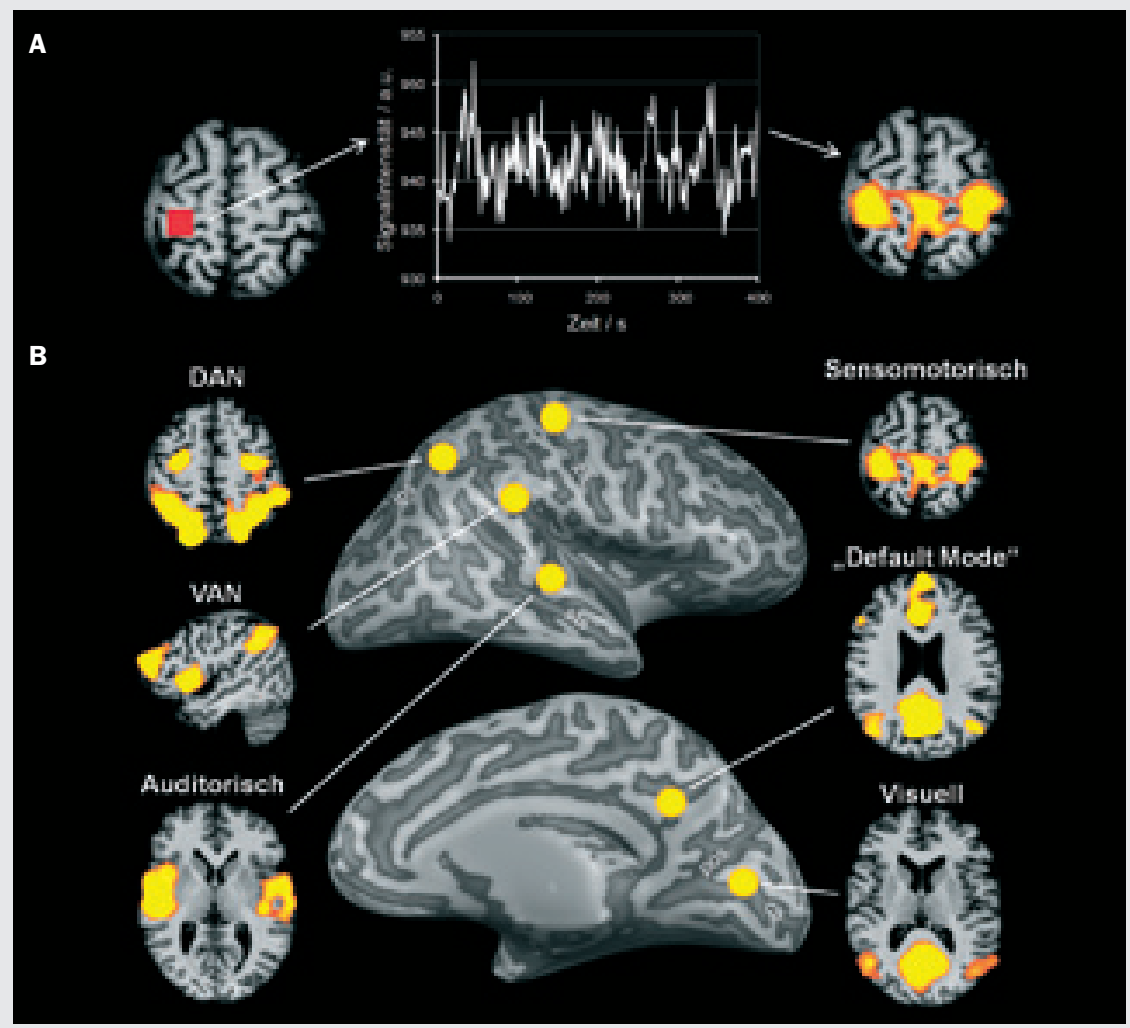

Abb. Exkurs: Ablauf und Ergebnisse einer funktionellen Konnektivitätsanalyse.

A) Grundsätzlicher Ablauf einer funktionellen Konnektivitätsanalyse am Beispiel des sensomotorischen Netzwerkes. Aus einer innerhalb des Netzwerkes relevanten Region (,Seed Region“), wie beispielsweise dem rechtshemisphärischen primären sensomotorischen Kortex (rotes Quadrat), wird der Signalintensitätszeitverlauf extrahiert. Dieser wird dann mit allen anderen Zeitverläufen korreliert und Regionen mit signifikant hohen Korrelationen werden farblich kodiert (gelb-orange). In der gezeigten axialen Schnittebene können so Kernregionen des sensomotorischen Netzwerkes, die bilateralen Repräsentationen des primären sensomotorischen Kortex sowie das supplementär motorische Areal, identifiziert werden.

B) Ergebnisse einer funktionellen Konnektivitätsanalyse bei einem Einzelprobanden. Auf der lateralen und medialen Ansicht der entfalteten rechten Hemisphäre sind die jeweiligen „Seed Regions“, aus denen die Zeitverläufe extrahiert wurden, markiert (gelbe Kreise). In den axialen Schnittebenen sind einige der jeweils identifizierten Kernregionen in verschiedenen primären sensorischen / motorischen sowie in höheren kognitiven Netzwerken dargestellt (gelb-orange). Unter anderem können die Repräsentationen des dorsalen (DAN) und ventralen (VAN, sagittale Schnittebene) Aufmerksamkeitsnetzwerkes kartiert werden. Abkürzungen: cs, calcariner Sulcus; ips, intraparietaler Sulcus; pos, parietookzipitaler Sulcus; sts, superiorer temporaler Sulcus; zs, zentraler Sulcus (Abb. adaptiert nach Raichle, 2011). 


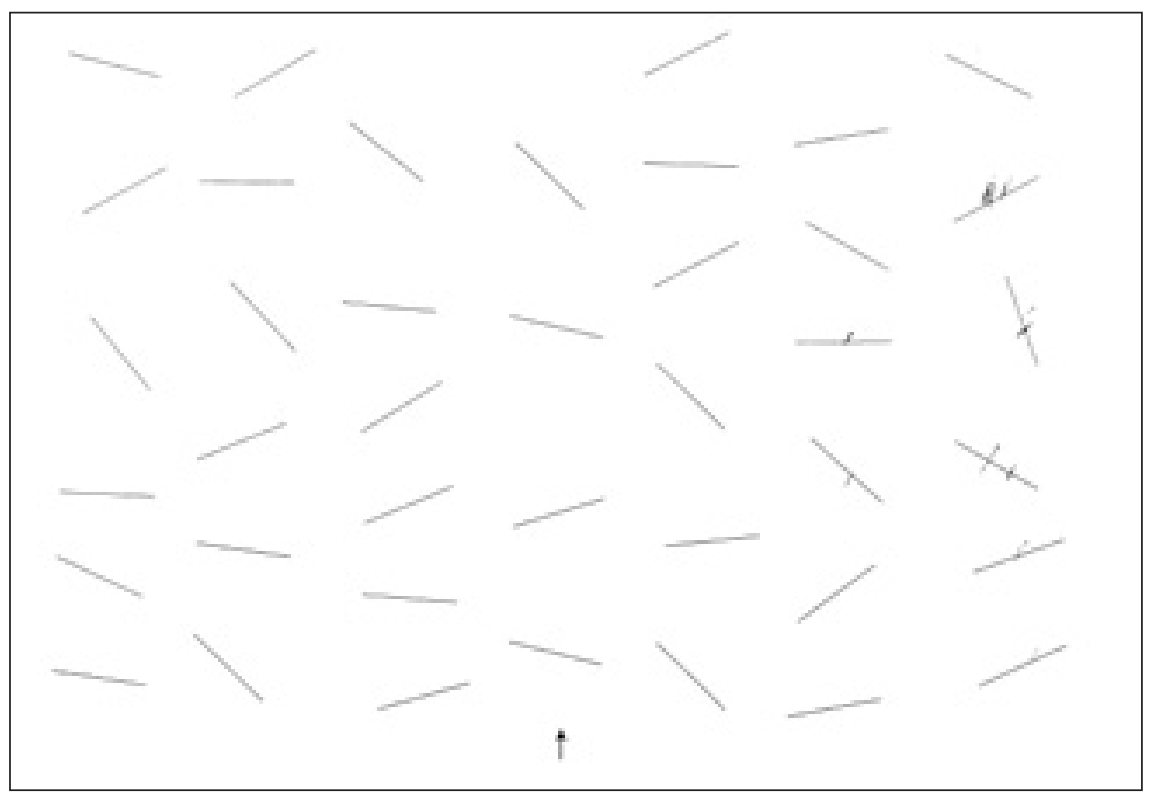

Abb. 1: Durchstreichaufgabe zur Neglect-Diagnostik. Der Patient hatte die Aufgabe, auf der DIN-A4-Vorlage ohne Zeitdruck alle Linien anzustreichen. Der Patient im akuten Stadium nach einem rechtshemisphärischen Insult lässt die linksseitig lokalisierten Striche aus und zeigt Perseverationsverhalten im rechten Halbfeld (siehe Mehrfach-Durchstreichungen).

genauere Verfahren wie die pharmakologische Inaktivierung von Hirnstrukturen verwendet werden.

\section{Räumliches Neglect-Syndrom}

Läsionen in Arealen, die im gesunden Gehirn bei der Ausführung räumlicher Aufgaben aktiviert werden, führen meist auch zu entsprechenden Verhaltensdefiziten. Dabei stellt das räumliche Neglect-Syndrom die häufigste Form dar, insbesondere dann, wenn die rechte Gehirnhälfte betroffen ist. Patienten mit Neglect verhalten sich so, als hätte die zur Läsion gegenüberliegende (kontraläsionale) Körper- oder Raumhälfte weitgehend aufgehört zu existieren. Dabei wird der kontraläsionale Raum nur unzureichend mit Kopf- und Blickbewegungen exploriert und sensorische Informationen werden in dieser Raumhälfte nur eingeschränkt verarbeitet (Abbildung 1). Häufig betrifft die Störung mehrere Sinnes-Modalitäten gleichzeitig und die Patienten ignorieren auf der kontraläsionalen Seite sowohl visuelle, auditorische als auch taktile Reize. Für die Patienten bedeutet das Auftreten eines Neglects eine ernsthafte Behinderung im Alltag, da sie mit auf der kontraläsionalen Seite gelegenen Gegenständen kollidieren, beim Lesen oder Schreiben Wörter auslassen und die betroffene Körperhälfte nicht mehr richtig einsetzen. Dabei lassen sich diese Defizite nicht aus Störungen der primären sensorischen oder motorischen Verarbeitung erklären (z.B. Gesichtsfeldausfälle, Halbseitenlähmung).

Es ist in diesem Kontext wichtig darauf hinzuweisen, dass Neglect ein Sammelbegriff für eine Vielzahl von Störungen darstellt und hinsichtlich Schweregrad und Ausprägung zwischen den Patienten eine beträchtliche Variabilität besteht. Viele Patienten zeigen anstatt eines voll ausgeprägten Neglects eine Vernachlässigung kontraläsionaler Reize nur, wenn diese simultan mit einem ipsiläsionalen Reiz präsentiert werden. $\mathrm{Ob}$ dieses als, räumliche Extinktion' bezeichnete Phänomen eine leichtere Form des Neglects oder eine eigenständige Störungsform darstellt, wird noch diskutiert, insbesondere weil die zugrunde liegenden Läsionsorte voneinander abweichen können (Bisiach 1991; Karnath et al. 2003; Karnath et al. 2011).

\section{Neglect-Modelle}

Zur Erklärung der diversen Neglectsymptome wurden verschiedene Erklärungsmodelle vorgeschlagen. Diese lassen sich grob in Aufmerksamkeits-, Repräsentations- und Transformationstheorien unterteilen. Während die Aufmerksamkeitstheorien davon ausgehen, dass dem Neglect eine pathologische Verschiebung der Aufmerksamkeit in den ipsiläsionalen Raum zugrunde liegt, unterstreichen die
Repräsentationstheorien, dass auch die interne, oder mentale Repräsentation des kontraläsionalen Raumes gestört ist. Transformationstheorien sehen im Neglect ein Defizit, räumliche Informationen zwischen verschiedenen Raum-Koordinatensystemen zu transformieren und postulieren eine Rotation der Körperwahrnehmung in Bezug zur visuellen Umgebung.

Das einflussreichste und am häufigsten empirisch getestete Neglect-Modell ist das des hemisphärischen/ AufmerksamkeitsUngleichgewichts. Dieses von Kinsbourne (1977) formulierte Modell beruht auf der Annahme, dass jede Gehirnhälfte einen Orientierungsvektor enthält, der die Aufmerksamkeit in das gegenüberliegende (kontralaterale) Halbfeld verschiebt. Während sich beide Gehirnhälften im Normalzustand wechselseitig inhibieren und damit eine dynamische Balance herstellen, kommt es bei Läsion in einer Gehirnhälfte zu einem Ungleichgewicht. Neglect entsteht demzufolge, weil die geschädigte Hemisphäre die intakte Hemisphäre nur unzureichend inhibieren kann, diese daraufhin eine Hyperexzitabilität entwickelt und sich als Konsequenz der Aufmerksamkeitsvektor in die ipsiläsionale Raumhälfte verschiebt. Mithilfe systematischer fMRT-Studien an NeglectPatienten und gesunden Probanden ist es inzwischen gelungen, die hemisphärische Ungleichgewichtstheorie zu erweitern und anatomisch zu spezifizieren. In der Literatur hat sich dabei die Annahme von zwei distinkten Aufmerksamkeitsnetzwerken durchgesetzt, einem dorsalen und ventralen (Corbetta und Shulman 2011). Das bilateral organisierte dorsale Aufmerksamkeitsnetzwerk (DAN) umfasst dabei die frontalen Augenfelder und Areale im intraparietalen Sulcus (IPS), während sich das ventrale Netzwerk (VAN) aus inferioren und mittleren Teilen des frontalen und temporoparietalen Kortex in der rechten Hemisphäre zusammensetzt. fMRT-Studien ergaben, dass das DAN auf die Aufmerksamkeitsfokussierung und die Kontrolle von Augenbewegungen spezialisiert ist, während das VAN die Neuorientierung des Aufmerksamkeitsfokus hin zu neuen, relevanten Stimuli ermöglicht. In der Neuformulierung des Modells entsteht ein Neglect durch die Kombination eines läsionsbedingten hemisphärischen Ungleichgewichtes zwischen den Regionen des DAN (direkt oder indirekt durch Läsionen im VAN) und die durch Läsionen im VAN resultierende Störung der Zuwendung auf Reize außerhalb des Aufmerksamkeitsfokus (Abbildung 2). 


\section{Spektrum Sachbücher \\ Bildungsfutter und Lesespaß in einem!}

- Aktueile Neverscheinungen

Wie viel erklärt uns die

Hirnforschung wirklich?

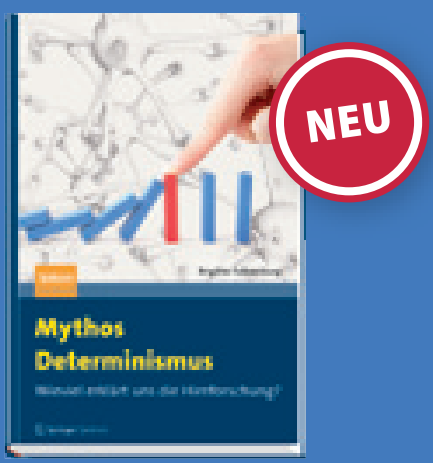

2012. 449 S. 15 Abb. geb. m. SU

ISBN 978-3-642-25097-2

$\triangleright €$ (D) $24,95 / €$ (A) 25,65 / * sFr 31,50

Brigitte Falkenburg

\section{Mythos Determinismus}

Aus der Sicht der Neurobiologie regiert im Kopf das neuronale Geschehen. Doch was wissen die Hirnforscher genau über die Mechanismen des Hirngeschehens und ihren Einfluss auf den menschlichen Geist? Dieser Frage geht Brigitte Falkenburg nach.

Die Physik hat sich längst vom mechanistischen Weltbild gelöst, in der Neurobiologie bleiben überholte mechanistische Vorstellungen bis heute wirksam. Dabei sind die „mechanistischen" Erklärungen der Hirnforschung ganz anders als ihr Name suggeriert; und wer annimmt, der Geist sei so strukturiert wie die Materie, zieht atomistische und kausale Fehlschlüsse über das Bewusstsein. Falkenburgs Buch möchte die Debatte um Geist und Gehirn, freien Willen und Determinismus endlich davon befreien. Es liefert Grundzüge einer Wissenschaftstheorie der Hirnforschung und eröffnet den Weg zu einem differenzierteren Naturverständnis und Menschenbild.

\section{- Rätsel Gehirn: Träumen, Denken, Fühlen

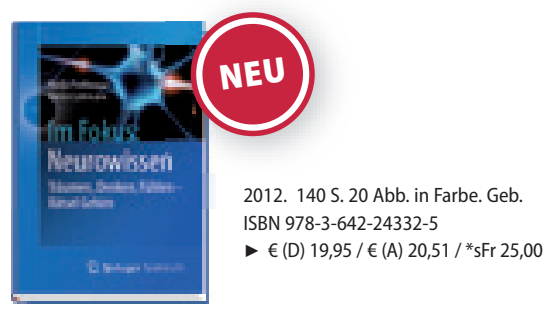

Nadja Podbregar / Dieter Lohmann

\section{Im Fokus: Neurowissen}

Können wir im Schlaf lernen? Wie weit reicht der Einfluss der Hormone? Und was bestimmt, ob wir hochbegabt oder nur Durchschnitt sind? Im Zentrum all dieser Fragen steht unser Gehirn, die Schaltzentrale unserer Persönlichkeit und unseres Menschseins. In den letzten Jahren sind Neurowissenschaftler immer tiefer in die Struktur und Physiologie des Gehirns vorgedrungen. Dieses Buch nimmt Sie mit auf Erkundungsreise zu faszinierenden, rätselhaften und beeindruckenden Phänomenen unseres Denkens, Fühlens und Bewusstseins.

\section{- Die Wahrheit über das Lügen}

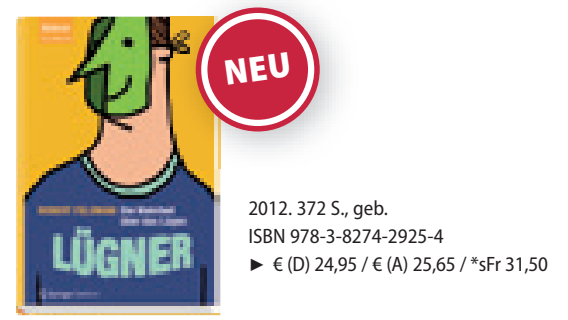

Robert S. Feldman

\section{Lügner}

Lügen, Täuschung und Unehrlichkeit spielen in unserem Zusammenleben eine erstaunlich große Rolle. Robert Feldman zählt zu den führenden Wissenschaftlern, die der Täuschung in ihren vielen Facetten auf den Grund gehen. Gespeist aus jahrelanger Forschungsarbeit und mit zahlreichen Beispielen aus dem Alltag bietet er in diesem Buch einen faszinierenden Einblick in eine große Macht in unserem Leben - die alltäglichen Täuschungen, an denen wir alle teilhaben. Denn jeder, der sagt, er erzähle immer die Wahrheit, ist ein Lügner.
Hirnforschung - Topaktueller Überblick

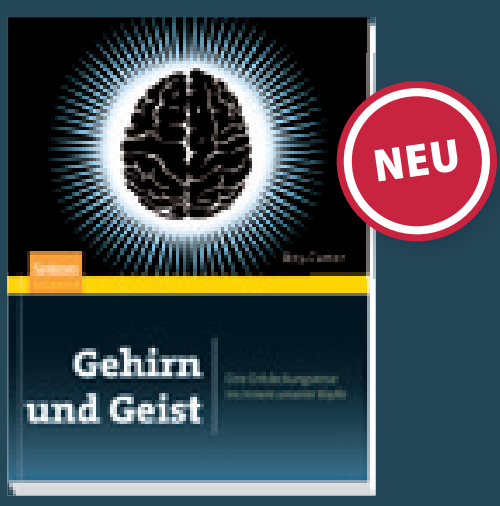

2012. 376 S. 140 Abb. in Farbe. Brosch.

ISBN 978-3-8274-2919-3

- $€(D) 24,95 / \epsilon$ (A) $25,65 / *$ sFr 31,50

Rita Carter

\section{Gehirn und Geist}

Verstehen wir unser Gehirn? Können die Forscher bereits eine präzise Landkarte des Geistes zeichnen? Wie gut ausgeleuchtet sind die Räume unserer Psyche? Und wo liegen die Grenzen unseres Wissens, die Geheimnisse, die wir noch ergründen müssen?

Die renommierte britische Wissenschaftsjournalistin Rita Carter vermittelt uns in der Neuauflage ihres vielgelobten Buches nicht nur einen spannenden Überblick über den aktuellen Stand der Hirnforschung, sondern gewährt uns auch außergewöhnliche Einblicke in die Organisation und Arbeitsweise unseres Gehirns - und liefert damit zugleich einen Schlüssel zum Verständnis von Geist und Bewusstsein.

"Einer der anschaulichsten und best illustrierten Versuche, das praktisch Unzugängliche zu erklären - das Gehirn."

Sunday Times

"Ein geradezu atemberaubendes Kompendium von Fallstudien, Forschungsergebnissen und Spekulationen. Carters Ansatz ist mutig und zeigt ein beeindruckendes Gespür für die Schwierigkeiten der Hirnforschung. Gehirn und Geist ist hervorragend, informativ und provokativ." New Scientist 


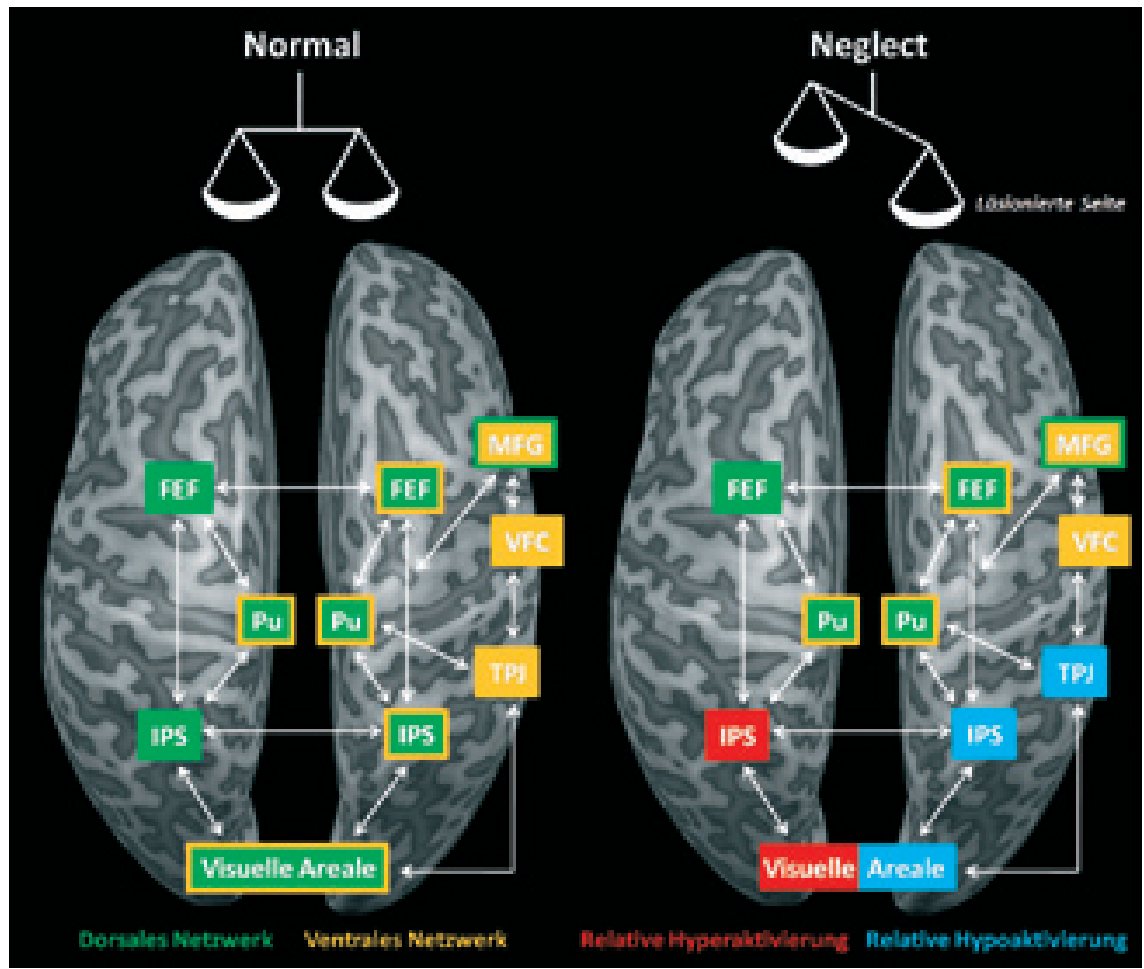

Abb. 2: Modell des dorsalen (DAN) und ventralen (VAN) Aufmerksamkeitsnetzwerkes beim gesunden Probanden (links) und beim Neglect-Patienten (rechts). Im gesunden Probanden bilden die Areale im DAN eine dynamische Balance. Das VAN ist beim Menschen in der rechten Hemisphäre dominant. Durch eine rechtshemisphärische Läsion kommt es zu einer Hypoaktivierung des rechten Parietalkortex und einer gleichzeitigen Hyperaktivierung des linken Parietalkortex. Dieses hemisphärische Ungleichgewicht kann entweder durch direkte oder indirekte Schädigung (zum Beispiel des VAN) hervorgerufen werden (Abbildung adaptiert und erweitert nach Vossel et al. 2010).

\section{Tiermodelle für räumlichen Neglect}

Für die Entwicklung effektiver diagnostischer und therapeutischer Ansätze ist ein fundamentales Verständnis der geschädigten Gehirnnetzkomponenten, ihrer spezifischen Funktionen und Interaktionen notwendig. Dies erweist sich bei neurologischen Patienten häufig als schwierig, da Ausmaß und Lokalisation der Läsionen zwischen den Patienten variabel sind und weil diese insbesondere im Akutstadium nach einem Schlaganfall häufig nicht in der Lage sind, über längere Zeit hinweg konzentriert kognitive Aufgaben durchzuführen. Gleichzeitig finden häufig schon in den ersten Wochen nach Hirnschädigung Reorganisationsprozesse in strukturell intakten Netzwerkanteilen sowie Verhaltenskompensationen statt, was die Unterscheidung zwischen Gehirnaktivität, die Funktionsausfällen bzw. ihrer Kompensation zugrunde liegt, erschwert. Deshalb sind experimentelle Modelle für das Testen spezifischer Hypothesen unverzichtbar.
Für die experimentelle Neglect-Forschung wurden sowohl Primaten- als auch Katzen- und Rattenmodelle entwickelt. Da vor allem nicht-humane Primaten in der Lage sind, komplexe kognitive Aufgaben zu lösen und ihr Gehirn die größte Homologie zum Menschen aufweist, dominieren diese die Neglect-Forschung. Die Primaten-Studien werden daher im Folgenden detaillierter behandelt.

\section{Strukturelle Läsionsstudien in nicht- humanen Primaten}

Neglect-Symptome wie ipsiläsionale Orientierungs- und Aufmerksamkeitstendenzen wurden beim Affen als Konsequenz einer Reihe von Läsionen in kortikalen und subkortikalen Strukturen beschrieben. Operationalisiert wurde der Neglect dabei meist als Tendenz, die Augen und den Körper spontan in den ipsiläsionalen Raum auszurichten, präferenziell auf der ipsiläsionalen Seite nach Futter zu suchen und als Beeinträchtigung bei der Detektion kontraläsionaler Reize.
Die ersten strukturellen Läsionsexperimente in nicht-humanen Primaten waren dabei vor allem auf die funktionelle Dissoziation von Arealen gerichtet, die bei humanen Neglect-Patienten häufig gleichzeitig geschädigt sind. Aus elektrophysiologischen Ableitungen beim Affen ist bekannt, dass der inferiore Parietalkortex aus diversen Subregionen besteht, deren Aktivität mit räumlicher Aufmerksamkeit, visuomotorischer Integration und der Steuerung von Augenbewegungen korreliert ist (Abbildung 3, Exkurs 2) (Clower et al. 2001).

Lynch und McLaren (1989) haben systematisch die Verhaltenseffekte von Läsionen im inferioren Parietalkortex und parietookzipitalen Kortex verglichen. Das Ausmaß des Neglects wurde als die Fähigkeit der Affen gemessen, mit den Augen einen Punkt zu verfolgen, der sich von der Mitte schnell nach links oder rechts bewegte. Dabei zeigte sich, dass weder Läsionen des inferioren Parietalkortex noch des parietookzipitalen Kortex einen Neglect für unilateral präsentierte Reize verursachten. Es fand sich aber eine Extinktion für kontraläsionale Reize, wenn diese gleichzeitig in beiden Gesichtsfeldern gezeigt wurden, sowie eine Verlängerung der Sakkaden-Latenzzeiten für kontraläsionale Reize. Lynch und McLaren schlossen aus diesen Beobachtungen, dass der parietale und parietookzipitale Kortex beim Affen mehr für die Initiierung und Kontrolle von sakkadischen Augenbewegungen wichtig ist und weniger für die Aufmerksamkeitssteuerung. Ein ähnliches Ergebnis wurde in einer Studie von Watson et al. (1994) berichtet. In dieser Studie zeigten die Affen nach Läsion des inferioren Parietalkortex zwar Störungen von zielgerichteten Arm- und Handbewegungen, aber keine Neglect-Symptomatik. Die Abwesenheit von Neglect nach Läsionen im inferioren Parietalkortex beim Affen ist überraschend, da diese Region beim Menschen für die Entstehung von Neglect kritisch zu sein scheint (Mort et al. 2003).

Im Gegensatz dazu wurde Neglect beim Affen beobachtet, wenn Teile des superioren temporalen Sulcus (STS) geschädigt wurden. Elektrophysiologische Studien ergaben, dass der STS aus einer Reihe von Arealen besteht, deren neuronale Aktivität mit visueller Aufmerksamkeit, multimodaler Integration und Augenbewegungssteuerung korreliert (Exkurs 2).

Diese und ähnliche Beobachtungen haben zu Diskussionen über die anatomischen und funktionellen Homologien des inferioren Parietalkortex beim Affen und Menschen geführt und zu der Hypothese, dass der STS beim Affen das funktionelle Homolog des 
inferioren Parietalkortex beim Menschen darstellt (Luh et al. 1986). Basierend auf dieser Debatte haben Karnath et al. (2001) Neglect-assoziierte Läsionen beim Menschen noch einmal genauer untersucht und gefunden, dass auch beim Menschen der kritische Läsionsort im oberen Temporallappen, und nicht wie zuvor angenommen im inferioren parietalen Kortex, liegt.

Generell lässt sich feststellen, dass Neglect-Symptome beim Affen sowohl hinsichtlich des Schweregrades als auch hinsichtlich ihrer Dauer hinter denen der humanen Patienten zurückbleiben. Ein weiterer Unterschied ist, dass Neglect beim Menschen vorwiegend nach Läsionen der rechten Hemisphäre auftritt, während beim Affen diese Form der Lateralisierung räumlicher Funktionen nicht beobachtet wurde. Bei der Erforschung der für den Neglect kritischen Läsionsorte ist die Interpretation struktureller Läsionen beim Affen ähnlich problematisch wie bei Patienten: unbeabsichtigte Schädigung der Faserverbindungen und einsetzende Reorganisationsprozesse. Eine wichtige Ergänzung stellen daher reversible Läsionsverfahren dar, die Neurone aber nicht ihre Verbindungsfasern inaktivieren.

\section{Reversible Läsionsstudien in nicht- humanen Primaten}

Die laterale Bank des intraparietalen Sulcus (LIP) ist das hinsichtlich Neglect am detailliertesten untersuchte Areal im Affen. LIP erhält anatomischen Input von extrastriatalen visuellen Arealen und ist reziprok mit dem FEF, TPO, den mittleren Schichten des SC und verschiedenen thalamischen Kernen, wie dem dorsalen Pulvinar verbunden. Neurone in LIP antworten auf visuelle Stimuli in ihrem rezeptiven Feld. Diese räumlich spezifischen visuellen Antworten werden sowohl durch die Richtung einer auszuführenden Augenbewegung als auch durch attentionale und motivationale Faktoren moduliert.

Wardak et al. (2001) inaktivierten beim Affen Neurone in LIP mittels lokaler Injektionen des GABA-A-Agonisten Muscimol und evaluierten die Konsequenzen auf attentionales und okulumotorisches Verhalten. Nach der Inaktivierung brauchten die Affen mehr Zeit, um in einer komplexen Suchaufgabe kontraläsionale Reize zu finden. Während LIP-Inaktivierung sakkadische Augenbewegungen zu einzeln präsentierten Zielreizen nicht beeinträchtigte, führten die Affen fast ausschließlich ipsiläsionale Sakkaden aus, wenn zwei Reize in gegenüberliegenden Halbfeldern präsentiert wurden. Ähnliche ipsiläsionale Entscheidungstendenzen nach LIP-Inaktivierung wurden gefunden, wenn die Affen die Positionen der Zielreize aus dem Gedächtnis abrufen sollten (Abbildung 4) (Wilke et al. 2012). Zusammengenommen legen diese Defizite nahe, dass LIP eine wichtige Rolle bei der visuell-attentionalen Selektion von Augenbewegungszielen spielt.

Im Gegensatz zu LIP ergaben Inaktivierungsexperimente des FEF deutliche Defizite in der Ausführung sakkadischer Augenbewegungen auch zu einzeln präsentierten Reizen. Hierbei fanden sich Positionsungenauigkeiten kontraläsionaler Sakkaden und ein Anstieg der Latenzzeiten. Interessanterweise berichtete eine spätere Studie, dass eine FEF-Inaktivierung auch Defizite in einer visuellen Suchaufgabe hervorrief, wenn keine Augenbewegung erforderlich war (Wardak et al. 2006). Daraus lässt sich schließen, dass das FEF für die Okulomotorik bedeutsam ist, aber auch zu selektiven Aufmerksamkeitsprozessen beiträgt.

\section{Exkurs 2}

\section{Anatomie und Funktion fronto-parietaler und subkortikaler Netzwerke beim Affen}

\section{Parietalkortex}

Sowohl beim Menschen als auch beim Affen unterteilt der Sulcus intraparietalis den superioren und den inferioren Parietalkortex. Beim Affen gehört die Area 7 zum inferioren Parietallappen und enthält Neurone, die auf visuelle Stimuli reagieren und durch Augenbewegungen und Aufmerksamkeitsanforderungen moduliert werden. Tiefer gelegene Neurone in der medialen Bank des Sulcus intraparietalis (IPS (MIP/PRR)) feuern besonders dann, wenn der Affe gezielte Armbewegungen plant oder ausführt, während Neurone in der lateralen Bank (LIP) auf Augenbewegungen spezialisiert sind. Der anteriore Anteil des IPS (AIP) ist an der Vorbereitung von Greifbewegungen beteiligt, und Neurone im Fundus (VIP) integrieren visuelle mit vestibulären Signalen. Eine Reihe weiterer Areale, deren neuronale Aktivität mit visueller Aufmerksamkeit, multimodaler Integration und Augenbewegungssteuerung korreliert ist, befinden sich im superioren temporalen Sulcus (STS). Insbesondere das an der oberen Bank des STS gelegene superior temporale polysensorische Areal (STP), einschließlich des temporoparietookzipitalen Areales (TPO), enthält Neurone, die auf Stimuli in mehreren sensorischen Modalitäten reagieren und in die Planung von Augenbewegungen involviert sind.

\section{Frontalkortex}

Aus Einzelzellableitungen beim Affen wissen wir, dass auch der frontale Kortex eine Reihe effektorspezifischer Subareale enthält. Visuelle und Augenbewegungsrelatierte Aktivität findet sich dabei in den frontalen und supplementären Augenfeldern (FEF und SEF) und im Sulcus principalis. Auch Neurone im ventralen und dorsalen prämotorischen Kortex werden durch attentionale Faktoren moduliert und antworten auf Reize in unterschiedlichen Modalitäten, sofern diese ein potenzielles Ziel für eine spätere Handlung darstellen.

\section{Pulvinar}

Sowohl der parietale als auch der frontale Kortex sind anatomisch mit einer Reihe subkortikaler Strukturen verbunden, welche für die attentionale Steuerung von
Augen- und Handbewegungen sowie für multimodale Integration wichtig sind. $\mathrm{Zu}$ diesen subkortikalen Strukturen gehört auch das Pulvinar. Das Pulvinar ist ein thalamischer Kernkomplex, der reziprok mit einer Vielzahl von kortikalen Arealen verbunden ist, die mit der Verarbeitung visueller und visiomotorischer Informationen befasst sind. Das Pulvinar lässt sich grob in einen ventralen und einen dorsalen Aspekt unterteilen. Der ventrale Teil hat reziproke Verbindungen $\mathrm{zu}$ einer Vielzahl visueller Areale und erhält Input aus den oberen Schichten des Superioren Colliculus (SC). Der dorsale Anteil des Pulvinars ist mit kortikalen Arealen verbunden, die visuell-räumliche Aufmerksamkeits- und Augenbewegungsfunktionen erfüllen, z.B. dem FEF, dem parietalen LIP und Arealen im STS, wie MT/MST und TPO. Ein großer Anteil der Neurone im ventralen und dorsalen Teil antwortet auf visuelle Reize und wird durch Aufmerksamkeit sowie den Grad der bewussten Wahrnehmung moduliert (Bender und Youakim 2001; Wilke et al. 2009). Weiterhin konnte gezeigt werden, dass Pulvinar-Neurone ihre Feuerraten während und nach sakkadischen Augenbewegungen ändern (Robinson et al. 1990). 


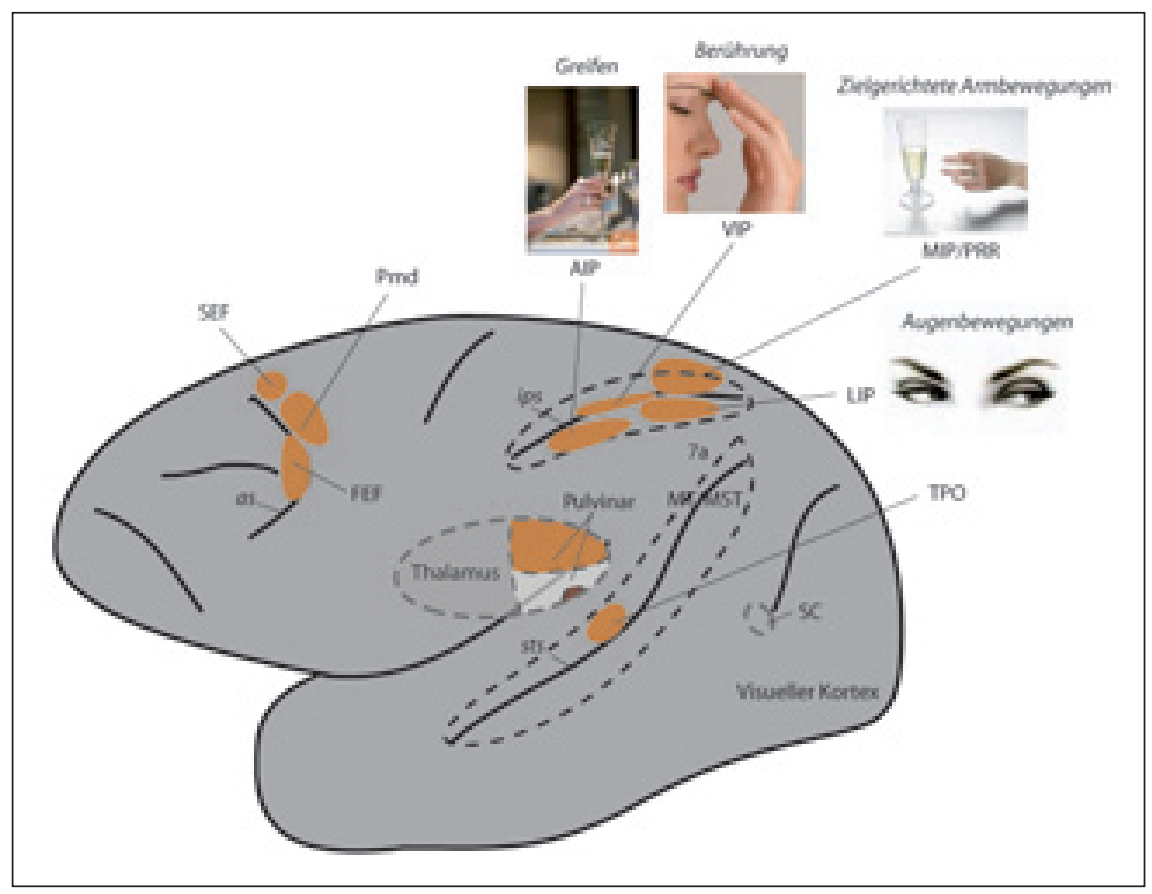

Abb. 3: Schematische Darstellung der für die räumliche Wahrnehmung und Handlungsplanung wichtigen Strukturen beim Rhesus Affen. Laterale Ansicht. Abkürzungen: AIP, anterior intraparietal; as, arcuate Sulcus; FEF, frontales Augenfeld; LIP, lateral intraparietal; MT, medial temporal; MST, medial superior temporal; Pmd, dorsaler prämotorischer Kortex; TPO, temporoparietookzipital; SC, Superiorer Colliculus; sts, superiorer temporaler Sulcus; ips, intraparietaler Sulcus; SEF, supplementäres Augenfeld. Zu beachten ist die funktionale Spezialisierung der im intraparietalen Sulcus gelegenen Areale.

Eine ähnliche Tendenz, ipsiläsionale $\mathrm{Ob}-$ jekte auszuwählen, wenn zwei Reize in gegenüberliegenden Halbfeldern gezeigt werden, wurde nach Inaktivierung des ventralen prämotorischen Kortex (PMv) gefunden. Neurone in diesem Areal antworten sowohl auf taktile Stimulation der oberen Extremitäten als auch auf sich bewegende visuelle Objekte in Armreichweite. Unilaterale Inaktivierung von PMv war dementsprechend mit einer Tendenz assoziiert, Hand- und Greifbewegungen in den ipsiläsionalen Raum auszurichten, ohne dass ein primäres motorisches Defizit erkennbar war (,Motor Extinktion“) (Schieber 2000).

Neglect-Symptome beim Affen wurden auch nach reversiblen Läsionen in subkortikalen Strukturen, wie dem dorsalen Pulvinar, berichtet (siehe Exkurs 2). Unilaterale Pulvinar-Inaktivierung führte $\mathrm{zu}$ Problemen beim Wechseln des Aufmerksamkeitsfokus ins kontraläsionale Halbfeld und zu Defiziten bei der Diskrimination visueller Reize (Petersen et al. 1987; Desimone 1990). Wir sind der Frage nachgegangen, ob Pulvinar-Inaktivierung die Planung und Ausführung von Augen- und Armbewegungen beeinflusst (Wilke et al.
2010). Dabei mussten die Affen entweder Bewegungen zu einzeln gezeigten Reizen ausführen oder konnten sich zwischen Reizen in gegenüberliegenden Halbfeldern entscheiden (Abbildung 4A). Nach der Inaktivierung waren die Affen zwar prinzipiell noch in der Lage, Augen- und Handbewegungen in alle Richtungen auszuführen, aber die Präzision war im kontraläsionalen Raum schlechter. Sowohl in der Augen- als auch in der Armbewegungsaufgabe zeigten die Affen eine ausgeprägte Tendenz, ipsiläsional gelegene Objekte zu wählen (Abbildung 4C). Gleichzeitig wurde auch die kontraläsionale Hand beim Greifen von Objekten nicht mehr spontan eingesetzt. Zusammengenommen verursachen dorsale Pulvinar-Läsionen also sowohl perzeptive als auch motorische Neglect-Symptome. Da die Verhaltenseffekte der PulvinarInaktivierungen den oben beschriebenen Konsequenzen von Läsionen in temporoparietofrontalen Arealen ähneln, wurden die Ergebnisse als sekundäre kortikale Dysfunktion interpretiert. Dies ist insofern plausibel, als der dorsale Teil des Pulvinars mit diesen kortikalen Netzwerken reziprok verbunden ist (Exkurs 2). Damit liegt das Pulvinar in einer strategischen Position, die
Aktivität innerhalb und zwischen diesen Arealen zu modulieren.

Um zu verstehen, welche Gehirnaktivitätsänderungen der Vernachlässigung des kontraläsionalen Raumes zugrunde liegen, haben wir im nächsten Schritt Teile des dorsalen Pulvinars inaktiviert und gleichzeitig fMRT-Antworten gemessen, während die Affen die oben beschriebene SakkadenAufgabe ausführten (Abbildung 5A, Exkurs 1). Wir verwendeten dabei ein ereignis-relatiertes (,event-related“) Design, um zwischen BOLD-Änderungen in verschiedenen Aufgabenperioden unterscheiden zu können (z.B. Fixation, Hinweisreiz, Sakkade). In Kontrollexperimenten ohne Inaktivierung führte die Präsentation von einzelnen Sakkaden-Hinweisreizen und die darauffolgende Gedächtnisperiode zur Aktivierung des FEF, verschiedener Areale im IPS (z.B. LIP) und des dorsalen STS (z.B. MST, TPO) (Abbildung 5B). Nach Inaktivierung des rechten Pulvinar fanden wir Aktivitätsänderungen in anatomisch verbundenen kortikalen Arealen. In den kortikalen Arealen auf der Seite der Injektion fanden wir eine Reduktion der BOLD-Aktivität in temporoparietalen Arealen, vor allem in LIP, MST und TPO (Abbildung 5B). Diese Reduktion war besonders ausgeprägt für Reize, die auf der kontraläsionalen Seite gezeigt wurden. Gleichzeitig fanden wir, dass Reize im ipsiläsionalen Halbfeld nun mehr Aktivität hervorriefen, insbesondere in der intakten linken Hemisphäre. Dieses Aktivitätsmuster ist im Prinzip mit der hemisphärischen Ungleichgewichtstheorie des Neglects vereinbar (Kinsbourne 1977). Die separate Auswertung der Aktivität für links versus rechts präsentierte Hinweisreize, ergab aber auch, dass die Aktivitätserhöhung in der intakten Hemisphäre kein generelles Phänomen darstellte, sondern spezifisch für ipsiläsionale Reize war.

Zusammenfassend lässt sich schlussfolgern, dass das dorsale Pulvinar bei der Verarbeitung räumlicher Informationen und bei der Handlungsplanung eine wichtige Rolle spielt, und dass Pulvinar-Läsionseffekte auf kortikale Netzwerkaktivität sowohl anatomisch- als auch Gesichtsfeldspezifisch sind. Da BOLD- und Zellaktivität dissoziiert sein können (Maier et al. 2008) und wegen der eingeschränkten zeitlichräumlichen Auflösung der fMRT (Exkurs 1), werden zukünftige elektrophysiologische Studien im Affen klären müssen, wie sich Pulvinar-Läsionen auf die neuronalen Antworteigenschaften in verbundenen kortikalen Arealen auswirken. Denkbar wäre eine Modulation der Antwort-Amplituden, der rezeptiven Feldeigenschaften oder der 


\section{Exkurs 3}

\section{Nicht-invasive Hirnstimulation}

Direkte, invasive Hirnstimulation zum Nachweis kausaler Zusammenhänge ist beim Menschen nur in Ausnahmefällen (z.B. intraoperativ während geplanter Eingriffe am Gehirn) möglich. In den letzten Jahrzehnten wurden allerdings verschiedene Verfahren etabliert, die eine elektrische Beeinflussung der Gehirnaktivität durch die intakte Schädeldecke hindurch erlauben.

In der neurowissenschaftlichen Forschung hat sich vor allem die transkranielle Magnetstimulation(TMS) durchgesetzt. Die TMS (Barker et al. 1985) beruht auf dem Prinzip der elektromagnetischen Induktion. Eine tangential über den Schädelknochen gehaltene Stimulationsspule wird kurzzeitig von einem starken elektrischen Strom durchflossen. Dabei entsteht kurzfristig ein starkes magnetisches Feld, das in der Lage ist, Gewebe wie die Schädelkalotte zu durchdringen. Durch die Änderung des magnetischen Feldes wird in leitfähigem Gewebe ein elektrisches Feld induziert, das in einem geeignet ausgerichteten Axon eine Depolarisation und ein Aktionspotenzial auslösen kann (vgl. Siebner und Ziemann
2007; Wassermann et al. 2008; Sandrini et al. 2011)

In den kognitiven Neurowissenschaften ist vor allem die Eigenschaft der TMS entscheidend, die normale Funktionsweise des Kortex zeitweise zu beeinträchtigen und damit eine funktionelle Läsion („virtual lesion“) zu verursachen. Während der Bearbeitung einer Aufgabe werden dazu einzelne oder kurze Serien von TMS - Pulsen über einem definierten Kortexbereich appliziert (online-TMS). Führt dies zu einer Beeinträchtigung der Verhaltensleistung (z.B. erhöhte Fehlerrate oder verlängerte Reaktionszeiten), so nimmt man an, dass das betreffende Kortexgebiet wesentlich für die untersuchte Funktion ist.

Da die zeitliche Auflösung der TMS exzellent ist (für Einzelpulse im Bereich von Hundertstelsekunden), können virtuelle Läsionen dazu benutzt werden, den genauen Zeitpunkt zu bestimmen, an dem ein Kortexareal an der Durchführung einer Aufgabe beteiligt ist (mentale Chronometrie). Problematisch für die Neurowissenschaften sind die räumlichen Eigenschaften der TMS: Aufgrund der Abschwächung des Magnetfeldes mit zunehmender Entfernung von der Spule sind die tieferen Hirnstrukturen (z.B. der Thalamus oder die Basalganglien) für die TMS nicht erreichbar. Das primäre Ziel der TMS ist der Kortex (Eindringtiefe ca. 1-3 cm). Die erreichbare räumliche Auflösung liegt für fokale Doppelacht-Spulen im Bereich von wenigen Quadratzentimetern.

Mit repetitiver TMS (rTMS) lassen sich neuronale und Verhaltenseffekte erzielen, die das Stimulationsende überdauern. Diese Eigenschaft der rTMS macht man sich bei offline-TMS-Experimenten zunutze.

Die transkranielle Gleichstromstimulation (tDCS) mit Oberflächenelektroden und vergleichsweise niedrigen Stromstärken um $1 \mathrm{~mA}$, wurde im letzten Jahrzehnt als weiteres Verfahren entwickelt, um Hirnfunktionen nicht-invasiv zu beeinflussen (vgl. Zaghi 2010). Gleichstromstimulation führt polaritätsabhängig zu Veränderungen der kortikalen Erregbarkeit: anodale tDCS steigert, kathodale tDCS vermindert die Erregbarkeit des Motorkortex. Der Effekt beruht dabei nicht auf der direkten Auslösung von Aktionspotenzialen wie bei der TMS, sondern in unterschwelligen modulatorischen Polarisationsveränderungen im neuronalen Gewebe, deren Wirkung ebenso wie bei der rTMS über die Dauer der Stimulation hinaus besteht. Die zeitliche und räumliche Auflösung der tDCS ist geringer als bei der TMS, die tDCS ist jedoch nebenwirkungsärmer und einfacher einzusetzen.
Synchronisation zwischen kortikalen Neuronen. Zusammenfassend sprechen die oben diskutierten Versuche in nicht-humanen Primaten prinzipiell für die Theorie des hemisphärischen Ungleichgewichtes, wobei sich dieses vor allem in Aktivitätsunterschieden zeigt, die durch kontra- versus ipsiläsionale Stimuli hervorgerufen werden. Experimente, welche spezifisch die neurobiologischen Grundlagen der Transformationstheorie testen, stehen nach unserem Wissen noch aus.

\section{Neglect beim Menschen: Störung attentionaler Netzwerke}

Räumlicher Neglect tritt beim Menschen nach Schädigung verschiedener Gehirnstrukturen auf, die untereinander anatomisch verbunden sind. Obwohl die für Neglect kritischen Läsionsorte noch diskutiert werden, besteht weitgehende Einigkeit darüber, dass Neglect nach Läsionen des parietalen Kortex insbesondere des inferioren parietalen Kortex (IPL) (Mort et al. 2003) und des oberen temporalen Kortex (STG) (Karnath 2001) vorkommt. Neglect-Symptome wurden aber auch nach Läsionen des Gyrus frontalis inferior (IFG) (Husain und
Kennard 1996) und der Inselregion berichtet. Außer nach Schädigungen in kortikalen Arealen tritt Neglect auch nach Läsionen in subkortikalen Strukturen auf, beispielsweise nach Schädigung der Basalganglien und des Pulvinar (Karnath 2005). Obwohl Neglect im Akutstadium sowohl nach links- als auch nach rechtshemisphärischen Läsionen vorkommt, ist er beim Menschen typischerweise schwerwiegender und persistenter nach rechtshemisphärischen Läsionen. Neben der Läsionslokalisation unterstreichen insbesondere neuere Studien die Bedeutung der funktionalen Integrität dieser aus einzelnen Arealen zusammengesetzten kortikalen und subkortikalen Netzwerke. Für den Netzwerkcharakter des Neglect spricht unter anderem, dass betroffene Patienten häufig Schädigungen in der weißen Substanz aufweisen (Urbanski et al. 2008), und dass elektrische Stimulation von Faserverbindungen zwischen parietalen und frontalen Regionen einen ipsiläsionalen Bias im für die Neglect-Diagnostik verwendeten Linienhalbierungstest hervorruft (Thiebaut de Schotten et al. 2005).

Gleichzeitig konnte mithilfe von fMRTStudien bei Neglect-Patienten gezeigt werden, dass akuter Neglect mit funk- tioneller Deaktivierung in strukturell intakten aber anatomisch verbundenen Arealen assoziiert war: Bei einer visuellen Aufmerksamkeitsaufgabe zeigten NeglectPatienten mit Läsionen im rechten temporoparietalen Kortex in der Akutphase eine funktionelle Deaktivierung in strukturell intakten frontoparietalen Arealen in der geschädigten Hemisphäre (Corbetta et al. 2005). Gleichzeitig fand sich noch robuste BOLD-Aktivität in homologen Arealen in der intakten Hemisphäre. Im Verlauf der Genesung erschien der rechte dorsale parietale Kortex re-aktiviert und die Aktivität im linken dorsalen parietalen Kortex war im Vergleich zur Akutphase niedriger. Diese Ergebnisse wurden als konsistent mit Kinsbournes Theorie des hemisphärischen Ungleichgewichts interpretiert.

Weitere Aufschlüsse über die Rolle fronto-parietaler Netzwerke ergab eine Studie von $\mathrm{He}$ et al. (2007). Hier wurde die funktionelle Konnektivität (siehe Exkurs 1) im ventralen (VAN) und dorsalen Aufmerksamkeitsnetzwerk (DAN) untersucht (Abbildung 2). Wie bereits oben erwähnt, ist das DAN auf die Aufmerksamkeitsfokussierung und sensomotorische Integration spezialisiert, während das 
A

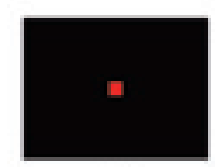

6.85

Fixation
Sakkaden-Entscheidungs-Aufgabe

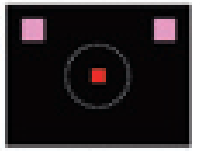

$200 \mathrm{~ms}$

HinweisReiz

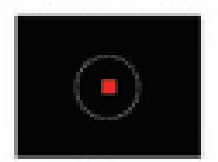

Ss

GedachtnisIntervall

C
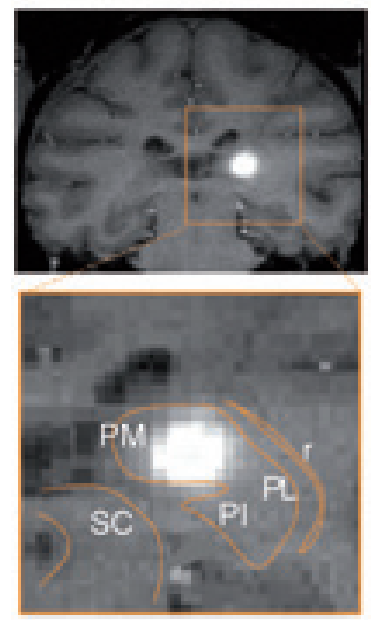

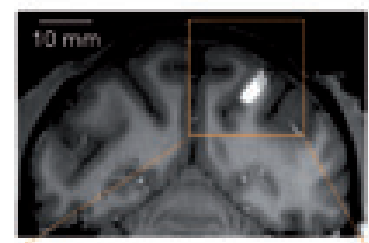

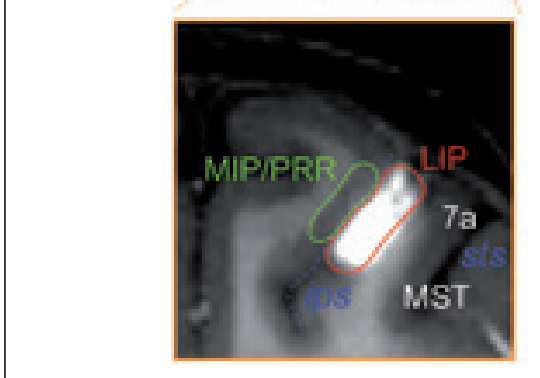

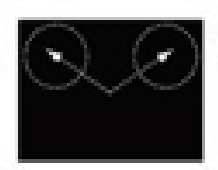

Sakkade
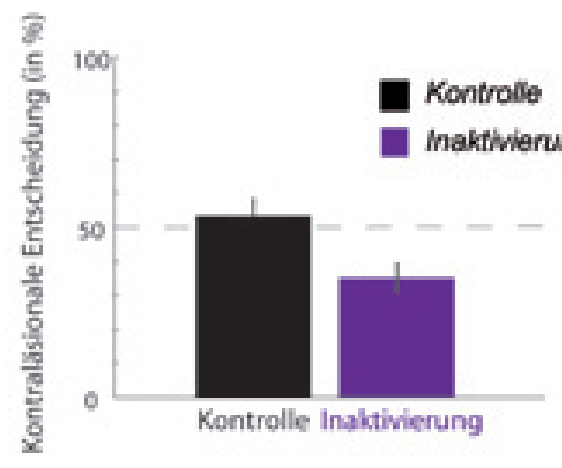

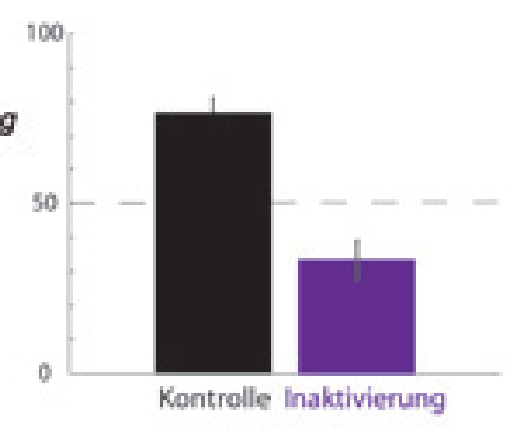

VAN die Neuorientierung des Aufmerksamkeitsfokus hin zu neuen, relevanten Stimuli ermöglicht. Neglect-Patienten mit Läsionen im VAN wiesen eine verminderte funktionelle Konnektivität zwischen strukturell intakten Arealen des DAN auf, genauer zwischen dem linken und rechten posterioren Parietalkortex. Dabei war eine stärkere Konnektivitätsreduktion mit einer schwereren Neglect-Symptomatik assoziiert. Im Gegensatz dazu resultierte eine direkte Schädigung parietaler Areale, bei strukturell intaktem VAN, nicht in einer schweren Neglect-Symptomatik (He et al. 2007). Zusammenfassend scheint also in diesen Fällen eine Schädigung des VAN und die daraus resultierende funktionale Beeinträchtigung des DAN für Neglect ursächlich zu sein (Corbetta und Shulman 2011).

\section{Reversible Neglectstudien beim Menschen}

Im Folgenden werden wir einige Studien vorstellen, bei denen mittels nicht-invasiver Hirnstimulation (TMS, tDCS: siehe Exkurs 3) Neglect-Symptome bei gesunden Probanden ausgelöst wurden. Hierbei haben wir Studien ausgewählt, in denen durch Beeinflussung der Funktion kortikaler Areale verschiedene Aspekte des hemisphärischen/ Aufmerksamkeits-UngleichgewichtsModelles untersucht wurden (Kinsbourne 1977).

In einer frühen Studie wurden die Auswirkungen einer kurzen Serie von TMS-Pulsen über verschiedenen kortikalen Arealen auf die Detektion visueller Reize untersucht, die entweder links, rechts oder
Abb. 4: Effekt von LIP- und Pulvinar-Inaktivierung auf Sakkaden-Entscheidungen.

A) Sakkaden-Entscheidungs-Aufgabe. Die Affen hatten die Aufgabe, mit den Augen einen in der Mitte des Bildschirmes präsentierten Punkt zu fixieren. Daraufhin wurden jeweils zwei Hinweisreize an randomisierten Positionen in gegenüberliegenden Halbfeldern präsentiert. Nach Verschwinden der Hinweisreize mussten die Affen diese Positionen im Gedächtnis behalten und nach einer Verzögerung von 5 sec dorthin eine Sakkade ausführen. Dabei konnten sie zwischen den beiden Positionen wählen und bekamen dieselbe Belohnung für eine richtige Sakkade in das linke oder rechte Halbfeld.

B) LIP-Inaktivierungsposition (oben) und Entscheidungsverhalten (unten).

Die Injektionsposition wurde im MRT mit der paramagnetischen Substanz Gadolinium sichtbar gemacht, welche wir zusammen mit dem GABA-A - Agonisten Muscimol injizierten (weißer Kontrast). Muscimol inaktiviert die Zellen im Injektionsareal bis zu mehreren Stunden. Wie in der untenstehenden Balken-Grafik zu sehen ist, hatte eine LIP - Inaktivierung eine Reduktion der kontraläsionalen Entscheidungen zur Folge (Wilke et al. 2012).

C) Dorsale Pulvinar-Inaktivierungsposition (oben) und Entscheidungsverhalten (unten). In Kontrollexperimenten zeigte dieser Affe eine Entscheidungspräferenz in Richtung des (späteren) kontraläsionalen Halbfeldes. Nach der Inaktivierung mit dem GABA-A - Agonisten THIP fand sich eine ausgeprägte Reduktion der kontraläsionalen Sakkaden-Entscheidungen. Abkürzungen: $r$, retikulärer thalamischer Kern; PI, inferiores Pulvinar; PL, laterales Pulvinar; PM, mediales Pulvinar.

bilateral präsentiert wurden (Pascual-Leone et al. 1994). Dabei zeigte sich, dass TMS über dem Parietalkortex eine Extinktion „kontraläsionaler" Stimuli bei bilateraler Präsentation induzierte. Vermehrte Fehler auch bei einzeln präsentierten Reizen, wie sie typisch für einen Neglect sind, konnten in dieser Studie mit parietaler Stimulation nicht erzielt werden, zeigten sich aber bei der okzipitalen Stimulation. Dies wurde von den Autoren jedoch nicht als Neglect, sondern als Beeinträchtigung des visuellen Kortex gesehen (,sensory detection block“). Im Unterschied zu den Befunden bei Patienten (aber ähnlich zu den oben beschriebenen parietalen Läsionsexperimenten beim Affen), konnte durch parietale TMS also kein vollständiger Neglect ausgelöst werden. Interessanterweise konnte auch keine 
Dominanz der rechten Hemisphäre für diese Aufgabe festgestellt werden.

In einer Folgestudie konnten Hilgetag et al. (2001) darüber hinaus nachweisen, dass inhibierende Stimulation über dem rechten Parietalkortex nicht nur die Detektion peripherer Stimuli im linken Halbfeld beeinträchtigte, sondern sich die Performanz im rechten Halbfeld verbesserte. Diese paradoxe Verbesserung einer Verhaltensleistung nach einem inhibierenden rTMS-Protokoll lässt sich gut mit dem Modell des hemisphärischen Ungleichgewichts nach Kinsbourne vereinbaren. Demnach führt eine Inhibition des rechten Parietalkortex zu einer verminderten transkallosalen Inhibition des linken Parietalkortex. Dieser wird dadurch übererregbar, was sich in einer verbesserten Detektionsleistung für die ipsiläsionalen, rechtsseitigen Reize zeigt.

In einer weiteren Studie wurde auch der Effekt gleichzeitiger bilateraler TMS getestet, welche aber keine Verschlechterung der Detektionsleistung zur Folge hatte (Dambeck et al. 2006). Auch dies kann als Hinweis darauf gedeutet werden, dass es das Aktivitätsungleichgewicht zwischen beiden Parietalkortices ist, welches eine Extinktion zur Folge hat.

Den Versuch, die von Kinsbourne postulierte Übererregbarkeit der linken Hemisphäre bei Neglect-Patienten mittels TMS direkt nachzuweisen, unternahmen Koch et al. (2008): Sie verwendeten dazu einen Aufbau mit zwei TMS-Spulen: Ein konditionierender TMS-Puls über dem Parietalkortex der gesunden Hemisphäre verstärkt die Muskelantwort auf einen Testpuls über dem gleichseitigen primären Motorkortex. Diese Verstärkung ist bei Neglect-Patienten stärker ausgeprägt als bei vergleichbaren Patienten mit ebenfalls rechtshemisphärischem Schlaganfall aber ohne Neglect. Zudem war linksparietale hemmende rTMS in der Lage, die Übererregbarkeit bei Neglect-Patienten zu normalisieren, und parallel dazu die Erkennungsleistung im kontraläsionalen Halbfeld zu verbessern.

In jüngster Zeit wurde gezeigt, dass auch mittels transkranieller Gleichstromstimulation (tDCS) über dem Parietalkortex eine Beeinflussung der räumlichen Aufmerksamkeit möglich ist (Sparing et al. 2009). In dieser Studie wurde berichtet, dass hemmende kathodale Stimulation die Detektion kontralateraler Stimuli beeinträchtigt, während anregende anodale Stimulation die Erkennung kontralateraler Stimuli verbessert. Bei Neglect-Patienten konnte sowohl durch kathodale Hemmung der gesunden Hemisphäre, als auch durch anodale Anregung der kontralateralen

\section{A} Sakkaden-Gedăchtnis-Aufgabe ('Instruierte Positionen')

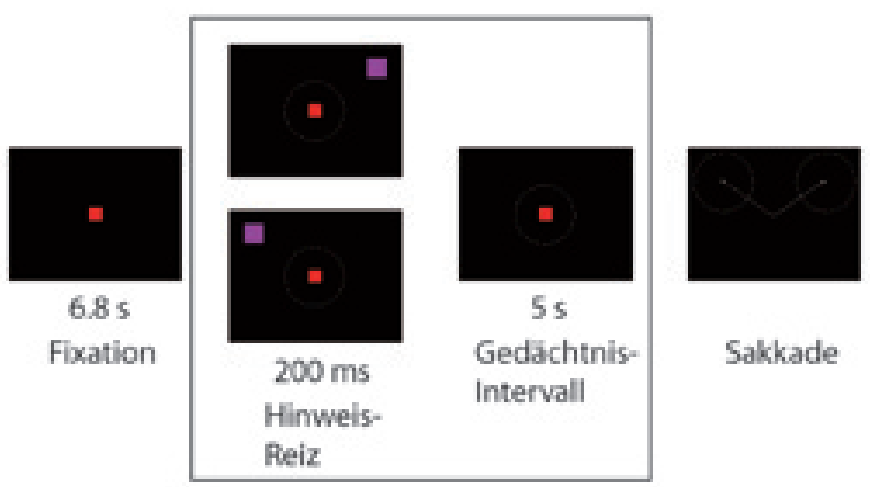

B

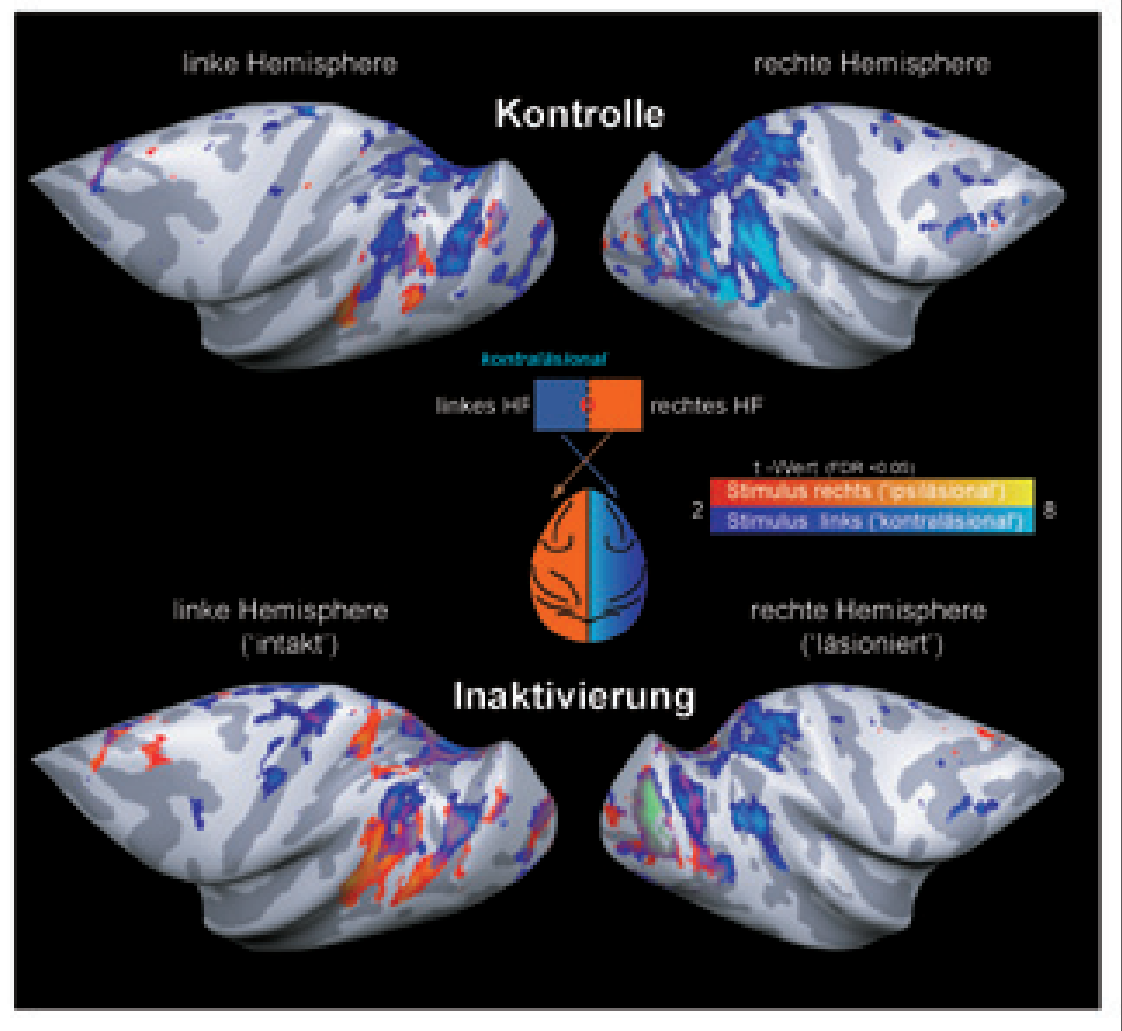

Abb. 5: Effekt von Inaktivierung des dorsolateralen Pulvinar auf BOLD-Aktivität für einzeln präsentierte sakkadische Hinweisreize.

A) Instruierte Sakkaden-Gedächtnis-Aufgabe. Die Affen hatten die Aufgabe, mit den Augen einen in der Mitte des Bildschirmes präsentierten Punkt zu fixieren. Daraufhin wurde ein Hinweisreiz an einer randomisierten Position im linken oder rechten Halbfeld präsentiert. Nach Verschwinden des Hinweisreizes mussten die Affen seine Position im Gedächtnis behalten und nach einer Verzögerung von 5 sec dorthin eine Sakkade ausführen. Der schwarze Rahmen zeigt die verwendete Zeitperiode für die Aktivitätskarten in (B).

B) Übereinander gelegte BOLD-Aktivitätskarten für links (blau) versus rechts (rot) präsentierte Hinweisreize vor (oben) und nach Inaktivierung (unten). In der inaktivierten Gehirnhälfte zeigte sich eine Reduktion der Aktivität für einzeln präsentierte Reize im kontraläsionalen (linken) Halbfeld (zu sehen als Reduktion der Intensität und Ausdehnung der Farbe Blau). Gleichzeitig fand sich ein Anstieg der Aktivierung für ipsiläsionale Hinweisreize (zu sehen als Anstieg der Intensität und Ausdehnung der Farbe Rot). Farbskala: t-Werte (von 2 bis 8). 


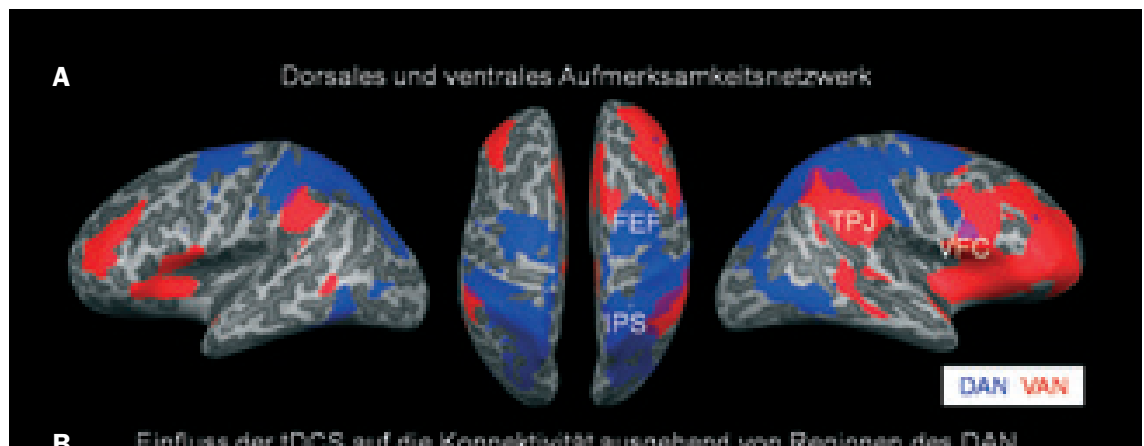

B Einfluss der tDCS auf die Konncktintat ausgehond won Regionen des DAN
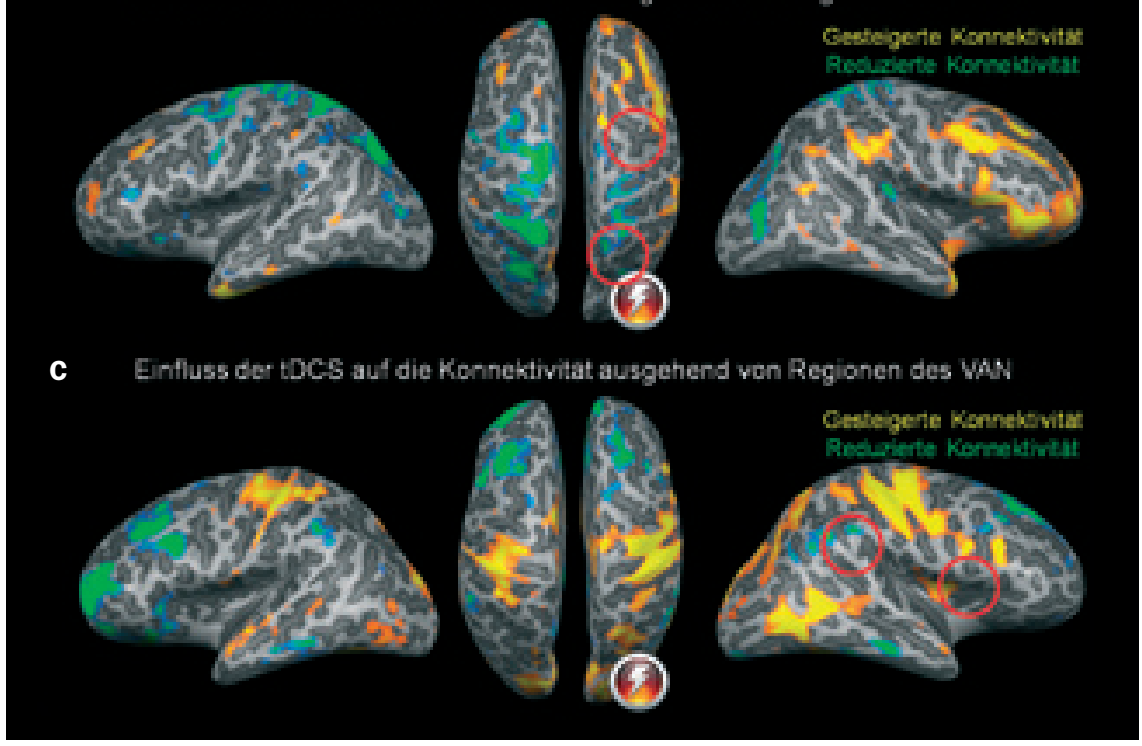

Abb. 6: Effekt anodaler tDCS über dem rechten IPS auf das ventrale und dorsale Aufmerksamkeitsnetzwerk.

A) Die Repräsentationen des rechts-lateralisierten ventralen (VAN, rot) und bilateralen dorsalen Aufmerksamkeitsnetzwerkes (DAN, blau) wurden durch eine funktionelle Konnektivitätsanalyse identifiziert. Rechtshemisphärische Startregionen zur Kartierung des DAN waren das frontale Augenfeld (FEF) und der intraparietale Sulcus (IPS), zur Kartierung des VAN, die temporoparietale Übergangsregion (TPJ) und der ventrofrontale Kortex (VFC). Die Netzwerke sind weitgehend separiert und zeigen nur wenig Überlappung (lila).

B) Vergleich der Konnektivitätskarten vor und nach 10 Minuten anodaler tDCS über dem rechten IPS (Blitz). Ausgehend von den Startregionen des DAN (rote Kreise) findet sich eine reduzierte Konnektivität (grün) innerhalb des DAN, aber eine gesteigerte Konnektivität (gelb) zwischen DAN und VAN nach tDCS.

C) Vergleich der Konnektivitätskarten vor und nach 10 Minuten anodaler tDCS über dem rechten IPS (Blitz). Ausgehend von den Startregionen des VAN (rote Kreise) findet sich eine reduzierte Konnektivität (grün) innerhalb des VAN, aber eine gesteigerte Konnektivität (gelb) zwischen VAN und DAN nach tDCS.

Hemisphäre die Neglect-Symptomatik bei einer Linienhalbierungsaufgabe verbessert werden.

Aufbauend auf diesen Vorarbeiten haben wir in eigenen Untersuchungen anodale tDCS und fMRT zunächst in gesunden Probanden kombiniert, um den Effekt parietaler Stimulation auf funktionelle Konnektivität im ventralen und dorsalen Aufmerksamkeitsnetzwerk zu testen. Wir fokussierten dabei auf Messungen der Ruheaktivität des Gehirns (,resting state”),
Inwieweit diese tDCS-induzierten Konnektivitätsveränderungen verhaltensrelevant sind, muss in Folgestudien noch geklärt werden.

\section{Offene Fragen und Ausblick}

Die Erforschung der neuronalen Grundlagen normaler und gestörter räumlicher Aufmerksamkeits- und Verhaltensprozesse hat in den letzen Dekaden große Fortschritte gemacht. Viele Fragen bleiben aber bisher noch unbeantwortet. Zum Beispiel ist noch nicht bekannt, inwieweit Läsionen in Arealen, deren Aktivität vor allem mit der Planung von Augen- oder Armbewegungen korreliert, auch effektorspezifische Neglect-Symptome hervorrufen. Im Affenmodell kann beispielsweise getestet werden, ob selektive Läsionen in LIP zu einer ipsiläsionalen Augenbewegungstendenz führen und Läsionen im angrenzenden MIP/PRR zu einer Armbewegungstendenz, aber nicht umgekehrt. Weiterhin ist die Frage der Homologien zwischen Menschen und Affen in dorsalen und ventralen Aufmerksamkeitsnetzwerken noch nicht abschließend geklärt. Es ist zu erwarten, dass vergleichende fMRT-Studien, die sowohl beim Menschen als auch am Affen durchgeführt werden können, zur Beantwortung dieser Frage wesentlich beitragen können. Voraussetzung ist allerdings, dass Menschen und Affen konsequent mit vergleichbaren und verfeinerten kognitiven Aufgaben getestet werden. Eine weitere offene Frage ist, inwieweit sich räumliche Aufmerksamkeits- und Wahrnehmungsdefizite von Handlungsdefiziten dissoziieren lassen. Da insbesondere die Mehrzahl der tierexperimentellen Studien Aufgaben verwendet haben, bei denen die Detektion von Objekten an eine gerichtete motorische Handlung gebunden waren, lässt sich diese Frage aus der Literatur bisher nicht zweifelsfrei beantworten. Notwendig wären Experimente, bei denen Wahrnehmung und Motorantwort dissoziiert werden, z.B. indem die Präsenz eines Objektes mit einer Bewegung in das gegenüberliegende Halbfeld oder mit einem Knopfdruck mit der ipsiläsionalen Hand angezeigt wird. Eine große Herausforderung für die nächsten Jahre wird es sein, die funktionalen Auswirkungen von Neglect-assoziierten Läsionen auf die neuronale Aktivität in verbundenen Netzwerkknoten zu verstehen und ein neurobiologisches Modell des Neglects zu entwickeln. Obwohl existierende Neglect-Theorien Annahmen über die zugrunde liegenden neuronalen Aktivitätsveränderungen formulieren, basieren diese 
auf Aktivitätsänderungen in Gehirnarealen und nicht auf der von Zellpopulationen. Es gibt zwar fMRT-Studien, welche Läsionseffekte auf entfernte, konnektierte Netzwerkkomponenten messen, aber die zugrunde liegenden zellulären Mechanismen sind unzureichend verstanden. Unklar ist daher, ob einer Änderung der BOLDAmplituden eine Veränderung der Anzahl und/oder Eigenschaften rekrutierter Neurone, der neuronalen Feuerraten oder Synchronisierung zwischen diesen zugrunde liegt. Eine Kombination aus reversiblen Inaktivierungen, fMRT- und elektrophysiologischen Studien beim Affen könnte zur Klärung dieser Fragen beitragen.

\section{Literatur}

Corbetta, M. und Shulman, G.L. (2011): Spatial neglect and attention networks. Annual review of neuroscience 34: 569-599.

Grefkes, C. und Fink, G.R. (2005): The functional organization of the intraparietal sulcus in humans and monkeys. Journal of anatomy 207: 3-17.

Kagan, I., Iyer, A., Lindner, A. und Andersen, R.A. (2010): Space representation for eye movements is more contralateral in monkeys than in humans. Proc Natl Acad Sci USA 107: 7933-7938.

Karnath, H.O. (2001): New insights into the functions of the superior temporal cortex. Nat Rev Neurosci 2: 568-576.

Kinsbourne, M. (1977): Hemi-neglect and hemisphere rivalry. Adv Neurol 18: 41-49.

Sparing, R., Thimm, M., Hesse, M.D., Kust, J., Karbe, H. und Fink, G.R. (2009): Bidirectional alterations of interhemispheric parietal balance by non-invasive cortical stimulation. Brain 132: 3011-3020.

Wilke, M., Kagan, I. und Andersen, R.A. (2012): Functional Imaging Reveals Rapid Reorganization of Cortical Activity after Parietal Inactivation in Monkeys. PNAS, in revision.

Wilke, M., Mueller, K.M. und Leopold, D.A. (2009): Neural activity in the visual thalamus reflects perceptual suppression. Proc Natl Acad Sci USA 106: 9465-9470.

Wilke, M., Turchi, J., Smith, K., Mishkin, M. und Leopold, D.A. (2010): Pulvinar inactivation disrupts selection of movement plans. The Journal of Neuroscience 30: 8650-8659.

Eine vollständige Literaturliste ist bei eForum, der englischen Online-Version bei Springer-Link zu finden.

\section{Danksagung}

Die Abteilung für Kognitive Neurologie wird durch die Universitätsmedizin Göttingen und die Hermann und Lilly Schilling-Stiftung finanziert. Die hier beschriebenen Arbeiten wurden durch die National Institutes of Health und die Schilling-Stiftung gefördert.

\section{Kurzbiografien}

Prof. Dr. Melanie Wilke studierte von 1997-2001 an der Ludwig-MaximiliansUniversität München (LMU) Psycholinguistik, Neuropsychologie und Neurobiologie. Im Anschluss promovierte sie bis 2005 am Max-Planck-Institut für Biologische Kybernetik (Tübingen). Danach war sie Postdoc am National Institute of Mental Health (Bethesda, USA) und am California Institute of Technology (Pasadena, USA). Seit 2011 leitet sie die von der Schilling-Stiftung eingerichtete Abteilung für „Kognitive Neurologie“ an der Universitätsmedizin Göttingen und wurde dort auf eine W3-Professur berufen. Gleichzeitig ist sie Gastwissenschaftlerin am Deutschen Primatenzentrum (DPZ, Göttingen), wo in Kooperation eine neue Arbeitsgruppe eingerichtet wurde. Der Arbeitsschwerpunkt der Abteilung liegt auf der Translation kognitiver Grundlagenforschung in gesunden Probanden und im Tiermodell auf klinische Fragestellungen.

PD Dr. Peter Dechent: Studium der Biologie in Mainz, Manchester, Stockholm und Göttingen. 2001 Promotion am Max-Planck-Institut für biophysikalische Chemie in Göttingen. Danach Postdoc in der Biomedizinischen NMR Forschungs GmbH. Seit 2004 Leiter der Forschungsgruppe „MR-Forschung in der Neurologie und Psychiatrie". 2009 Habilitation im Fach „Experimentelle Neuroradiologie“" an der Universitätsmedizin Göttingen. Seit 2011 ist die Forschungsgruppe in der Abteilung „Kognitive Neurologie“ unter Leitung von Prof. Dr. Wilke in der Universitätsmedizin Göttingen angesiedelt. Forschungsschwerpunkte sind die Charakterisierung der hämodynamischen Prozesse, welche der fMRT zugrunde liegen, sowie die Kombination nicht-invasiver Hirnstimulationsverfahren und fMRT mit dem Ziel, funktionelle Netzwerke zu charakterisieren und zu modulieren. Hier stehen insbesondere Veränderungen in den Aufmerksamkeitsnetzwerken bei Patienten mit Neglect-Syndrom im Fokus.

Carsten Schmidt-Samoa (M.A.): Studium der Humanmedizin (1989-1997), dt. Philologie, Philosophie und Wissenschaftsgeschichte (bis 2004) in Göttingen und Irvine, USA. Seit 2005 wissenschaftlicher Mitarbeiter der Universitätsmedizin Göttingen (Psychiatrie, Neurologie, Medizinische Psychologie). Seit 2008 Mitarbeiter der „MR-Forschung in der Neurologie und Psychiatrie“. Arbeitsschwerpunkte: Unter- suchung der Auswirkungen nicht-invasiver Hirnstimulation mit fMRT und Studium der Veränderungen visueller Aufmerksamkeit bei Neglect - Patienten mittels fMRT.

\section{Korrespondenzadressen}

Prof. Dr. Melanie Wilke

Abteilung Kognitive Neurologie

Universitätsmedizin Göttingen (UMG)

Robert-Koch-Str. 40

37075 Göttingen

Tel.: $\quad+495513913140$

Fax: $\quad+495513913243$

E-Mail:melanie.wilke@med.uni-goettingen.de

\section{PD Dr. Peter Dechent}

Abteilung Kognitive Neurologie

Universitätsmedizin Göttingen (UMG)

Robert-Koch-Str. 40

37075 Göttingen

Tel.: $\quad+495513913141$

Fax: +495513913243

E-Mail:pdechen@gwdg.de

\section{Carsten Schmidt-Samoa}

Abteilung Kognitive Neurologie

Universitätsmedizin Göttingen (UMG)

Robert-Koch-Str. 40

37075 Göttingen

Tel.: $\quad+495513913134$

Fax: $\quad+495513913243$

E-Mail: carsten.schmidt-samoa@med.unigoettingen.de 